\title{
Optimal use of methotrexate: the advantages of tight control
}

\section{Johannes W J Bïlsma, Michael E Weinblatt}

The CAMERA study shows that using methotrexate in a tight control setting might lead to considerable improvement in disease activity in early rheumatoid arthritis

\author{
See linked article p 1443
}

$\mathrm{T}$ he outlook for patients who are in 2007 newly diagnosed with rheumatoid arthritis has improved dramatically. ${ }^{1}$ Important elements in this improvement are: (1) the concept of a "window of opportunity": treat early and treat effectively; ${ }^{2}$ (2) early use of disease modifying anti-rheumatic drugs (DMARDs); ${ }^{3}$ (3) combination treatment, including glucocorticoids; ${ }^{45}(4)$ the use of biologicals; ${ }^{6}$ and (5) optimising therapy to the individual patient.

The CAMERA study reported in this issue by the Utrecht group (see page 1443) deals with this last item: optimising therapy to the individual patient. ${ }^{7}$ In this randomised controlled trial the drug treatment given to patients with early rheumatoid arthritis was the same in both groups, but the frequency and tightness of control were different. This report has some important messages that merit further comment:

1. The goal of tight control is feasible with the anchor drug methotrexate in a much larger proportion of patients than previously thought.

2. Using the same drug, but seeing the patient more often, and thus being able to adjust that specific drug more often, is much more efficacious then seeing the patient less frequently.

3. In a busy practice, tight control, aided by a computer-assisted protocol, is feasible.

\section{TIGHT CONTROL}

There is, as yet, no definition of "tight control" in rheumatoid arthritis. Elements of tight control are:

- seeing the patient frequently, to enable frequent adjustments to the therapy;

- improvement is not enough: the aim is low disease activity, or preferably even remission;

- being as objective as possible in the judgment of disease activity; not the doctor's or patient's impression, but an objective measurement of different aspects of disease activity, such as joint scores, visual analogue scale for well being, acute phase reactant in the blood;

- being rigorous in adjusting therapyfor example, a DAS of 1.7 is not 1.6; do not wait, but increase treatment according to protocol (a computer program may help in this respect);

- seeing the patient frequently until disease activity is under control.

\section{CORRECT DOSE OF METHOTREXATE}

As a definition of tight control we might therefore suggest that it is a treatment strategy for the individual rheumatoid arthritis patient, aiming for a predefined level of low disease activity or remission, within a limited period of time (as soon as possible). ${ }^{8}$

Six years ago a remarkable observation was made in the Netherlands when the first TNF- $\alpha$ blocker became available. There were serious concerns at government level regarding the costs of this new treatment. Therefore the Dutch Society for Rheumatology developed guidelines for when to use TNF- $\alpha$ blockers: patients needed first to be treated with at least two DMARDs in adequate dosages, including methotrexate, in a dosage of $25 \mathrm{mg} /$ week for at least three months before they could qualify for a TNF- $\alpha$ blocker. When estimates were made, it was thought that about $10 \%$ of the rheumatoid arthritis patients would need to be treated with TNF- $\alpha$ blockers; however, when methotrexate dosage was increased in order to qualify for this new treatment, many more patients responded to the higher methotrexate dosage. In addition, it also proved possible to increase the dosage of methotrexate quite rapidly when patients and doctors were anxious to reach that suggested higher dosage. In the first years only half of the expected number of rheumatoid arthritis patients were treated with TNF- $\alpha$ blockers, indicating that the potency of methotrexate was underused.

As reported in this issue, the CAMERA study $^{7}$ is a good example of optimising methotrexate treatment in early rheumatoid arthritis. Patients with early (disease duration $<$ l year) rheumatoid arthritis were randomly assigned to the intensive strategy or to the conventional strategy group. Both groups received methotrexate, the aim of treatment being remission. The intensive strategy group was seen every month and treatment was tailored to the individual patient on the basis of predefined response criteria, using a computerised decision program. Patients in the conventional group came to the outpatient clinic once every three months and were treated according to common practice. By increasing the dosage of methotrexate in the intensive strategy group monthly, a dosage of $30 \mathrm{mg} /$ week was reached within four months, leading to a much higher percentage of patients with low disease activity than previously reported. Remarkably, the tolerance of methotrexate at these higher dosages was comparable with that of lower dosages, apart from liver toxicity (23.2\% vs $18.6 \%$ ). In the study period of two years $50 \%$ of patients in the intensive strategy versus $37 \%$ of patients in the conventional strategy group achieved at least one period of remission, lasting at least three months. As expected, the difference was most pronounced in the first year (35\% vs $14 \%)$.

The correct dose of methotrexate is a critical issue to maximise control of disease activity in rheumatoid arthritis. Even though dose ranging studies with methotrexate in rheumatoid arthritis are limited, there is sufficient evidence that the effective dose for most patients is around $10 \mathrm{mg} / \mathrm{m}^{2}$.

In randomised controlled studies in which the methotrexate dose was escalated to $20 \mathrm{mg} /$ week, the effects on signs and symptoms of rheumatoid arthritis (as measured by the ACR 20 response criteria) were not statistically different between anti-TNF therapy as monotherapy (etanercept or adalimumab) and methotrexate $20 \mathrm{mg} /$ week at 6-12 months..$^{9-11}$

\section{AIMING FOR REMISSION}

On both sides of the Atlantic discussions are ongoing about how to define remission of rheumatoid arthritis in the $21 \mathrm{st}$ century. ${ }^{12}{ }^{13}$ Components of this definition are low disease activity, or even absence of disease activity, and no radiographic progression. There are of course good arguments to include no radiographic progression in an operational definition of remission. However it is far from practical to use a definition 
including radiographs for the day-to-day management of patients with early rheumatoid arthritis. But what about the use of disease activity scores in individual patients? For instance, the widely-used DAS is validated for groups but not for individual patients. When defining a patient in remission, the DAS has more face validity than the DAS28, just because the feet are not scored in the latter. Different components of the DAS have different influence on the total score, and in the lower range the influence of erythrocyte sedimentation rate is much greater than in the higher range. However, when evaluating a patient to decide whether treatment should be adapted, the patient's own perception of pain can be a major issue, especially when a pain syndrome has developed. Patients can score a DAS $>2$, just based on their pain perception, even though the doctor treating them sees no disease activity. When this leads to an increase in treatment-for example, by starting a TNF- $\alpha$ blocker-over-treatment might happen. Other aspects, such as regression to the mean of the DAS, might also be relevant in interpreting changes in the DAS. ${ }^{14}$ Clearly, more work needs to be done to define the best way to evaluate individual patients' remission or low disease state in such a way that individual treatment adaptations can be advised.

In the CAMERA study the reported cumulative dosage of methotrexate was comparable in both the conservative and intensive strategy group, which is remarkable. One explanation seems to be that because remission was quite often reached in the intensive strategy group, the dosage of methotrexate could be reduced in a substantial number of patients. It is also relevant to note that the cumulative use of NSAIDs was lower in the intensive strategy group. With regard to radiological progressions, the cumulative probability plot showed that in both strategy groups approximately $50 \%$ of patients did not progress over two years, but for the other $50 \%$ the progression rate was higher in the conventional strategy group than in the intensive strategy group. If the study protocol prescribed that the methotrexate dosage should not be decreased when remission was reached, perhaps this difference in radiographic progression would have been even more pronounced. This finding suggests that tight control is a good concept in bringing the disease under control, but that to maintain this control other aspects also need to be taken into account.

The CAMERA study clearly shows that using methotrexate in a tight control setting might lead to considerable improvement in disease activity in early rheumatoid arthritis. A programme to increase the dose of methotrexate to 20-25 mg/week in a more rapid manner will also lead to earlier identification of patients who are partial responders on methotrexate and might benefit from combination therapy. Tight control seems to be a promising new paradigm for reaching the aim of low disease activity-or, even better, remission in the first two years of treatment.

Ann Rheum Dis 2007;66:1409-1410.

doi: 10.1136/ard.2007.076463

\section{Authors' affiliations}

J W J Bïlsma, Department of Rheumatology \&

Clinical Immunology, UMC Utrecht, Utrecht, the Netherlands

M E Weinblatt, Department of Rheumatology and Immunology, Brigham \& Women's Hospital, Boston, MA, USA

Correspondence to: Professor J W J Biilsma, UMC Utrecht, PO Box 85500, 3508 GA Utrecht, the Netherlands; j.w.j.bij|sma@umcutrecht.nl

Accepted 24 August 2007

Competing interests: None declared.

\section{REFERENCES}

1 O'Dell JR. The BeSt way to treat early rheumatoid arthritis? Ann Intern Med 2007;146:459-60.
2 O'Dell JR. Treating rheumatoid arthritis early: a window of opportunity? Arthritis Rheum 2002;46:283-5.

3 VanderHeide A, Jacobs JW, Biilsma JW, Heurkens AH, VanBooma-Frankfort $C$, VanderVeen $M J$, et al. The effectiveness of early treatment with "second-line" antirheumatic drugs. A randomized controlled trial. Ann Intern Med 1996; 124:699-707.

4 Mottonen T, Hannonen P, Leirisalo-Repo $M$, Nissila $M$, Kautiainen $\mathrm{H}$, Korpela $M$, et al. Comparison of combination therapy with single-drug therapy in early RA: a randomized trial. FIN-RACo trial group. Lancet 1999;353:1568-73.

5 van Everdingen AA, Jacobs JW, Siewertsz van Reesema DR, Bijlsma JW. Low dose prednisone therapy for patients with early active rheumatoid arthritis: clinical efficacy, disease-modifying properties, and side effects: a randomized, doubleblind, placebo controlled clinical trial. Ann Intern Med 2002;136:1-12.

6 Smolen JS, Aletha D, Koeller M, Weisman MH, Emery P. New therapies for treatment of rheumatoid arthritis. Lancet, 2007 Jun 12 [Epub ahead of print].

7 Verstappen SSM, Jacobs JWG, van der Veen MJ, Heurkens AHM, Schenk Y, ter Borg EJ, et al. Intensive treatment with methotrexate in early rheumatoid arthritis: aiming for remission. Computer Assisted Management in Early Rheumatoid Arthritis (CAMERA, an open-label strategy trial). Ann Rheum Dis 2007;66:1443-9.

8 Bakker FM, Jacobs JWG, Verstappen SSM, Bijlsma JWJ. Tight control in the treatment of rheumatoid arthritis: efficacy and feasibility. Ann Rheum Dis 2007 (in press).

9 Bathon JM, Martin RW, Fleischman RM, Tesser JR, Schiff MH, Keystone EC, et al. A comparsion of etanercept and methotrexate in patients with early RA. N Engl J Med 2000;343:1586-93.

10 Klareskog $L$, vander Heijde $D$, de Jager JP, Gough A, Kalden J, Malaise M, et al. Therapeutic effect of the combination of etanercept and methotrexate compared with each treatment alone in patients with RA: double blind randomised controlled trial. Lancet 2004;363:675-81.

11 Breedveld FC, Weisman MH, Kavanaugh AF, Cohen SB, Pavelka K, van Vollenhoven R, et al. The PREMIER study: a multicenter, randomized, double-blind clinical trial of combination therapy with adalumimab plus methotrexate versus methotrexate alone or adalumimab alone in patients with early aggressive RA who had no previous methotrexate treatment. Arthritis Rheum 2006;54:26-37.

12 Aletaha D, Smolen JS. Remission of rheumatoid arthritis: should we care about definitions? Clin Exp Rheum 2006:24:S45-51.

13 Felson DT, Furst DE, Boers M. Rationale and strategies for reevaluating the ACR 20. J Rheumatol 2007:1184-7.

14 Greenwood MC, Rathi J, Hakim AJ, Scott DL, Doyle DV. Regression to the mean using the DAS in eligibility and response criteria for prescribing TNF alpha inhibitors in adults with RA. Rheumatology 2007;46:1165-7. 